OPEN ACCESS

Edited by:

Diego Franco,

University of Jaén, Spain

Reviewed by:

Carlos Martinez-Salgado,

University of Salamanca, Spain

Weichun $\mathrm{He}$

Second Affiliated Hospital, Nanjing

Medical University, China

*Correspondence:

Xiaoyan Wu

wxynjmu@163.com

${ }^{\dagger}$ These authors have contributed equally to this work

Specialty section:

This article was submitted to

Molecular Medicine

a section of the journa

Frontiers in Cell and Developmental

Biology

Received: 24 October 2019

Accepted: 20 March 2020

Published: 17 April 2020

Citation:

Mao L, Liu L, Zhang T, Qin H,

Wu $X$ and XU Y (2020) Histone

Deacetylase 11 Contributes to Renal

Fibrosis by Repressing KLF15

Transcription

Front. Cell Dev. Biol. 8:235.

doi: 10.3389/fcell.2020.00235

\section{Histone Deacetylase 11 Contributes to Renal Fibrosis by Repressing KLF15 Transcription}

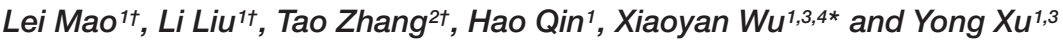 \\ 1 Key Laboratory of Targeted Intervention of Cardiovascular Disease and Collaborative Innovation Center for Cardiovascular \\ Translational Medicine, Department of Pathophysiology, Nanjing Medical University, Nanjing, China, ${ }^{2}$ Department of Geriatric \\ Nephrology, Jiangsu Province Hospital, First Affiliated Hospital of Nanjing Medical University, Nanjing, China, ${ }^{3}$ Institute \\ of Biomedical Research, Liaocheng University, Liaocheng, China, ${ }^{4}$ The Laboratory Center for Basic Medical Sciences, \\ Nanjing Medical University, Nanjing, China
}

Renal fibrosis represents a key pathophysiological process in patients with chronic kidney diseases (CKD) and is typically associated with a poor prognosis. Renal tubular epithelial cells (RTECs), in response to a host of pro-fibrogenic stimuli, can trans-differentiate into myofibroblast-like cells and produce extracellular matrix proteins to promote renal fibrosis. In the present study we investigated the role of histone deacetylase 11 (HDAC11) in this process and the underlying mechanism. We report that expression levels of HDAC11 were up-regulated in the kidneys in several different animal models of renal fibrosis. HDAC11 was also up-regulated by treatment of Angiotensin II (Ang II) in cultured RTECs. Consistently, pharmaceutical inhibition with a smallmolecule inhibitor of HDAC11 (quisinostat) attenuated unilateral ureteral obstruction (UUO) induced renal fibrosis in mice. Similarly, HDAC11 inhibition by quisinostat or HDAC11 depletion by siRNA blocked Ang II induced pro-fibrogenic response in cultured RTECs. Mechanistically, HDAC11 interacted with activator protein 2 (AP-2 $\alpha$ ) to repress the transcription of Kruppel-like factor 15 (KLF15). In accordance, KLF15 knockdown antagonized the effect of HDAC11 inhibition or depletion and enabled Ang II to promote fibrogenesis in RTECs. Therefore, we data unveil a novel AP-2 $\alpha$-HDAC11-KLF15 axis that contributes to renal fibrosis.

Keywords: renal fibrosis, renal tubular epithelial cell, transcriptional regulation, epigenetics, histone deacetylase

\section{INTRODUCTION}

Chronic kidney disease (CKD) is defined as progressive loss of key renal functions owing to a host of etiological factors including hypertension and diabetes (Coresh, 2017). For a large fraction of patients diagnosed with CKD, a transition to end-stage renal disease (ESRD) and renal failure is inevitable. Without effective long-term renal replacement therapy, prognosis for these patients is poor and mortality rate is high (Liu, 2013). Regardless of its etiology, ESRD is invariably preceded by renal fibrosis, characterized by accumulation of extracellular matrix (ECM) proteins in the renal interstitia, inflammatory infiltrates, proliferation and migration of myofibroblasts, and disruption 
of normal renal micro-architecture (Zeisberg and Neilson, 2010). Strong evidence suggests that there is a correlation between the severities of renal fibrosis and the outcome of ESRD (Nishitani et al., 2005). Thus, renal fibrosis can be considered as a common end-point for most kidney diseases (Gewin, 2018).

Investigations in the past decade have demonstrated that activated myofibroblasts are the unequivocal effector cell type and driver of renal fibrosis (Falke et al., 2015). Compared to quiescent fibroblasts, myofibroblasts possess a musclelike contractile phenotype and display much augmented proliferative and migratory ability. There remains great controversy with regard to the origin of myofibroblasts in the fibrotic kidneys. For instance, Humphreys et al. have shown, using the FOXD1-Cre driven tracing mice, that almost $100 \%$ of myofibroblasts are derived from pericytes in the unilateral ureteral obstruction (UUO) model and in the ischemia-reperfusion injury model of renal fibrosis (Humphreys et al., 2010). Zeisberg et al. (2007) have shown that up to $35 \%$ of myofibroblasts in the kidneys originate from endothelial cells whereas LeBleu et al. have reported that endothelial cells may account for no more than $10 \%$ of all myofibroblasts in the kidneys (Zeisberg et al., 2007; LeBleu et al., 2013). This apparent discrepancy stems from the different lineage-tracing tools that have been used in different studies. The evasiveness of "true" myofibroblast identities in vivo notwithstanding, many cell types, including renal tubular epithelial cells, fibroblast cells, and endothelial cells, can be induced to adopt a myofibroblast-like phenotype in vitro by transforming growth factor (TGF- $\beta$ ), platelet derived growth factor (PDGF-BB), angiotensin II (Ang II), and high glucose (Edeling et al., 2016). Accompanying the transition to myofibroblasts, profound changes in cellular transcriptome is taking place. The underlying epigenetic mechanism, however, is not clear.

The epigenetic machinery plays a key role in regulating mammalian cell transcription. It is generally considered that transcription status can be annotated by different histone modifications. Actively transcribed chromatin is abounded by acetylated histones whereas transcriptionally silenced chromatin is demarcated by low levels of acetylated histones and high levels of methylated H3K9 and H3K27 (Jenuwein and Allis, 2001). Histone acetylation and deacetylation are catalyzed by acetyltransferases and deacetylases, respectively. Traditionally, histone deacetylases (HDACs) fall into one of the four major categories: Class I HDACs, which include HDAC1, HDAC2, HDAC3, and HDAC8; Class II HDACs, which include HDAC4, HDAC5, HDAC6, HDAC7, HDAC9, and HDAC10; Class III HDACs, which include the sirtuin family of $\mathrm{NAD}^{+}$-dependent deacetylases; and Class IV HDAC, which contains a sole member HDAC11 (Seto and Yoshida, 2014). Whereas previous studies have demonstrated a role for class I HDACs (Liu et al., 2013; Choi et al., 2016), class II HDACs (Xiong et al., 2019), and class III HDACs (Morigi et al., 2018) in renal fibrosis, little attention has been paid to HDAC11 in this process. In the present study we investigated the role of HDAC11 in renal fibrosis. We report that HDAC11 expression is up-regulated in the fibrotic kidneys in mice and in Ang II-treated tubular epithelial cells in vitro. HDAC11 mediates Ang II induced profibrogenic response in tubular epithelial cells by interacting with AP- $2 \alpha$ to repress the transcription of KLF15. Therefore, targeting this AP-2 $\alpha$-HDAC11-KLF15 axis may yield novel therapeutic solutions against renal fibrosis.

\section{MATERIALS AND METHODS}

\section{Animals}

All animal experiments were review and approved by the Intramural Committee on Ethical Conduct of Animal Experiments. To induce renal fibrosis, the unilateral ureteral obstruction (UUO) procedure was performed in the mice. Briefly, a flank incision was made and the left ureter was ligated with silk suture at two points and cut between the ligatures. After the surgery, the animals were injected peritoneally with quisinostat $(100 \mathrm{mg} / \mathrm{kg}$, Selleck, Cat\# S1096) or vehicle twice a week until the day of sacrifice. The mice were sacrificed 14 days after the surgery. Alternatively, the mice were fed a high-fat diet (HFD, D12492, Research Diets) for 16 weeks as previously described (Xu et al., 2015). In the third model of renal fibrosis, the mice were implanted subcutaneously a minipump (Alzet 2004) that chronically released Ang II ( $1 \mu \mathrm{g} / \mathrm{kg} / \mathrm{min})$ for 4 weeks.

\section{Histology}

Histological analyses were performed essentially as described (Liu et al., 2018; Zhang et al., 2018b). Paraffin sections were stained with hematoxylin and eosin (Sigma), picrosirius red (Sigma), or Masson's trichrome (Sigma) according to standard procedures. Pictures were taken using an Olympus IX-70 microscope. Quantifications were performed with Image Pro.

\section{Cell Culture, Plasmids, Transient Transfection, and Reporter Assay}

Immortalized human renal tubular epithelial cells (HK-2, ATCC) and HEK293 cells were maintained in DMEM supplemented with $10 \%$ fetal bovine serum (FBS, Hyclone). Primary tubular epithelial cells (Xu et al., 2015), primary podocytes (Murakami et al., 2010), and primary renal fibroblast cells (Kunzel et al., 2019)were isolated as previously described. HDAC11 expression plasmids (Watanabe et al., 2014), HDAC11 promoter-luciferase constructs (Voelter-Mahlknecht et al., 2005), and KLF15 promoter-luciferase constructs (Shao et al., 2018) have been previously described. Small interfering RNAs were purchased from Dhamarcon. Transient transfections were performed with Lipofectamine 2000 (Invitrogen). Cells were harvested $48 \mathrm{~h}$ after transfection and reporter activity was measured using a luciferase reporter assay system (Promega) as previously described (Yang et al., 2018).

\section{Protein Extraction and Western Blot}

Before harvesting, cells were washed twice with ice-cold PBS buffer. Cell pellet was obtained by spinning in a refrigerated centrifuge at 2,500 rpm for $10 \mathrm{~min}$. Supernatant was discarded and cells was lysed in ice-cold RIPA buffer (1xPBS, $0.1 \%$ 
SDS, $1 \%$ NP-40, $0.5 \%$ sodium deoxycholate) supplemented with $100 \mu \mathrm{g} / \mathrm{ml}$ PMSF plus one protease inhibitor tablet (Roche, Mannheim, Germany) per $10 \mathrm{ml}$ RIPA buffer as previously described (Li et al., 2018d; Yang et al., 2019a,b; Zhang et al., 2019). Typically, 50-100 $\mu \mathrm{g}$ of proteins were loaded and separated by $8 \%$ PAGE-SDS gel with all-blue protein markers (Bio-Rad). Proteins were transferred to nitrocellulose membranes (Bio-Rad) in a Mini-Trans-Blot Cell (Bio-Rad). The membranes were blocked with $5 \%$ fat-free milk powder in Tris-buffered saline at room temperature for half an hour and then incubated with the following primary anybodies at $4^{\circ} \mathrm{C}$ overnight: anti-CTGF (Proteintech, 23936-1), anti-HDAC11 (Abcam, ab166907), anti- $\alpha$-SMA (Sigma, A5228), anti-KLF15 (Abcam, ab2647), anti-AP-2 $\alpha$ (Abcam, ab52222), and anti- $\beta$ actin (Sigma, A2228) antibodies.

\section{RNA Isolation and Real-Time PCR}

RNA was extracted with a commercial RNAprep purification kit (Tiangen) as previously described (Shao et al., 2019; Weng et al., 2019). First-strand synthesis was carried out using a HiScript III RT SuperMix (Vazyme). Realtime PCR reactions were performed on an ABI Prism StepOne Plus system with a commercial Sybrgreen kit (Vazyme) using the following primers: mouse Hdac11, 5' TTACAACCGCCACATCTACC- $3^{\prime}$ and $5^{\prime}$-GACATTCCTC TC CA CCTTCT C-3'; mouse Acta2, 5'-CTGAGCGTGGCTATT CCTTC- $3^{\prime}$ and reverse $5^{\prime}$-CTTCTGCATCCTGTCAGCAA- $3^{\prime}$; mouse Col1a1, 5'-GACGCCATCAAGGTCTACTG- $3^{\prime}$ and $5^{\prime}$-AC GGGAATCCA-TCGGTCA-3'; mouse Colla2, 5'-GCCACCAT TGATAGTCTCTCC- $3^{\prime}$ and $5^{\prime}$-CACCCCAGCGAAGAACT CATA-3'; mouse Col3a1, 5'-GGAACCTGGTTTCTTCTCACC$3^{\prime}$ and $5^{\prime}$-AGGACTGACCAAGGTGGCT-3' ${ }^{\prime}$; mouse $T g f b, 5^{\prime}$-GG AGAGCCCTGGATACCAAC- $3^{\prime}$ and $5^{\prime}$-CAACCCAGGTCCT TCCTAAA- $3^{\prime}$; mouse Klf15, 5' $5^{\prime}$-CCAATGCCGCCAAACC TAT- $3^{\prime}$ and $5^{\prime}$-GAGGTGGCTGCTCTTGGTGTACATC- ${ }^{\prime}$; mouse $C c l 2,5^{\prime}$-GAAGGAATGGGTCCAGACAT- $3^{\prime}$ and $5^{\prime}$-ACG GGTCAACTTCACATTCA-3'; mouse $I l-1 b, 5^{\prime}$-GCACTACAGG CTCCGAGATGAAC- $3^{\prime}$ and 5'-TTGTCGTTGCTTGGTTC TCCTTGT-3'; mouse Il-6, 5' -CCAGCTATGAACTCCTTCTC-3' and $5^{\prime}$-GCTTGTTCCTCACATCTCTC-3'; human HDAC11, $5^{\prime}$-ACCCAGACAGGAGGAACCATA- $3^{\prime}$ and 5'-TGATGTCC GCATAGGCACAG-3'; human COL1A1, 5'-AGGCGAACA GGGCGACAGAG-3' and $5^{\prime}$-GGCCAGGGAGACCGTTGAGT3'; human ACTA2, 5'-CATCCTCCCTTGAGAAGAGTTA-3' and $5^{\prime}$-TACATAGTGGTGCCCCCTGATA- $3^{\prime}$; human CTGF, 5'-GTTTGGCCCAGACCCAACT-3' and 5'-GGAACA GGCGCTCCACTCT-3'; human KLF15, 5' -AGCAAGGACTTG GATGCCTG-3' and 5'-AGGGCAGGTTCAAGTTGGAG-3'.

\section{Chromatin Immunoprecipitation}

Chromatin Immunoprecipitation (ChIP) assays were performed essentially as described before (Fan et al., 2017, 2019; Li et al., 2017, 2018a,b,c,e, 2019; Yu et al., 2017, 2018; Zeng et al., 2018; Zhang et al., 2018a; Kong et al., 2019a,b; Li and Xu, 2019; Liu et al., 2019a,b). In brief, chromatins were cross-linked with $1 \%$ formaldehyde for $15 \mathrm{~min}$ at room temperatures. Cells were incubated in lysis buffer $(150 \mathrm{mM} \mathrm{NaCl}, 25 \mathrm{mM}$ Tris $\mathrm{pH} 7.5$,
$1 \%$ Triton X-100, 0.1\% SDS, 0.5\% deoxycholate) supplemented with protease inhibitor tablet and PMSF. DNA was fragmented into $\sim 500$ bp pieces using a Diagenode Bioruptor sonicator. For each ChIP reaction, $100 \mu \mathrm{g}$ of protein were incubated at $4^{\circ} \mathrm{C}$ overnight with $2 \mu \mathrm{g}$ of the following antibodies: anti-HDAC11 (Abcam, ab166907), anti-AP-2 $\alpha$ (Abcam, ab52222), anti-acetyl H3 (Millipore, 06-599), and anti-acetyl H4 (Millipore, 06-598). For Re-ChIP, immune complexes were eluted with the elution buffer (1\% SDS, $100 \mathrm{mM} \mathrm{NaCO3}$ ), diluted with the Re-ChIP buffer (1\% Triton X-100, 2 mM EDTA, $150 \mathrm{mM} \mathrm{NaCl}, 20 \mathrm{mM}$ Tris $\mathrm{pH} 8.1$ ), and incubated with a second antibody of interest. Precipitated DNA was amplified with the following primers: KLF15 promoter \#1, 5'-AGCGAGCTGCGGGCGGGCT-3' and 5'-ACTCTCGGTCCGGCCGGC-3'; KLF15 promoter \#2, 5' -AA GCAAGGAGGTGGCT- $3^{\prime}$ and $5^{\prime}$-AAGGCTCGCAGGAGGCT3'; KLF15 promoter \#3, 5'-AAACCTCCTTAGTCCTG-3' and $5^{\prime}$-AGTGTCAGATAAATCACTTG-3'; KLF15 promoter \#4, $5^{\prime}$-AGCACCGTCAGCCCACGTG- $3^{\prime}$ and $5^{\prime}$-AGTGTCAGATAA ATCACTTG-3'; KLF15 promoter \#5, 5'-AGACCTGCACT GAGAC-3' and 5'-AGAGGCTTTCTATTC-3'; GAPDH promoter, 5' -GGGTTCCTATAAATACGGACTGC-3' and 5'-CTGGCACTGCACAAGAAGA-3'.

\section{Immunofluorescence Staining}

For immunofluorescence staining, paraffin sections were permeabilized with $0.1 \%$ Triton X-100 in PBS for $10 \mathrm{~min}$ and then blocked with 5\% BSA for $20 \mathrm{~min}$ at room temperature followed by incubation with anti-CD3 (BD Biosciences, 1:500) or anti-CD45 (BD Biosciences, 1:500) overnight. The nuclei were counterstained with DAPI (Sigma). 3 slides were stained from each individual mouse and $\sim 5$ fields were counted per slide. The data are presented as the relative number of positive cells/field.

\section{Statistical Analysis}

One-way ANOVA with post hoc Scheff'e analyses were performed by SPSS software (IBM SPSS v18.0, Chicago, IL, United States). $P$ values less than 0.05 were considered statistically significant.

\section{RESULTS}

\section{HDAC11 Is Up-Regulated by Pro-fibrogenic Stimuli in vivo and in vitro}

We first made an attempt to establish a relationship between the expression levels of HDAC11 with renal fibrosis in both animal models and cell models. In the first model, C57/BL6 mice were subjected to the unilateral ureteral obstruction (UUO) procedure. Compared to the sham mice, the UUO mice displayed up-regulation of HDAC11 mRNA paralleling elevation of $\alpha$ SMA, which correlates with the maturation of myofibroblasts, and fibronectin, a component of the extracellular matrix, in the kidneys (Figures 1A,B). In the second model, C57/BL6 mice were fed with a high-fat diet for 16 weeks to induce renal fibrosis. Quantitative PCR and Western blotting analyses revealed that HDAC11 levels were much higher in the HFD-fed kidneys than in the control-fed kidneys (Figures 1C,D). In the third model, 
A

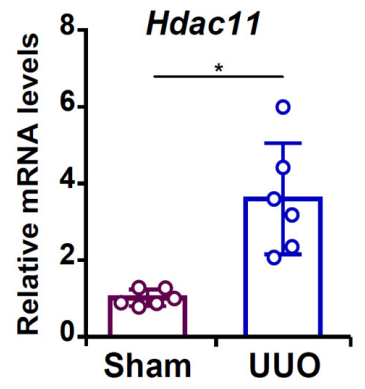

C

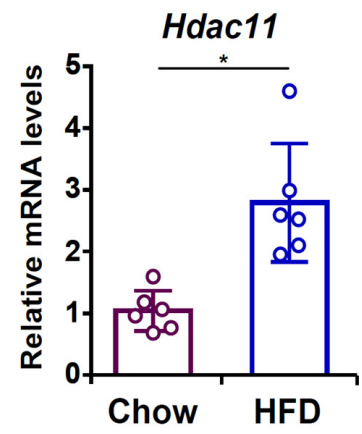

E

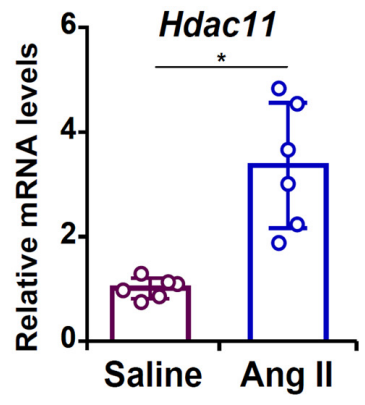

G

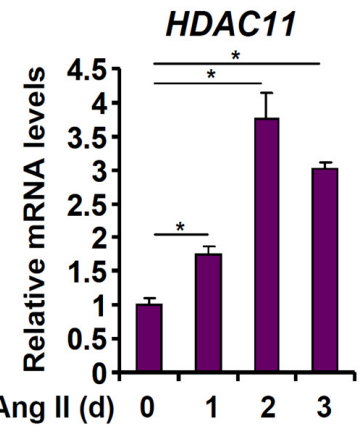

B
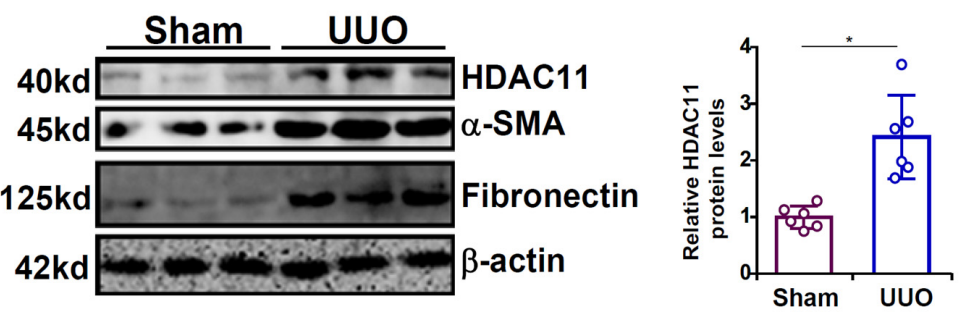

D
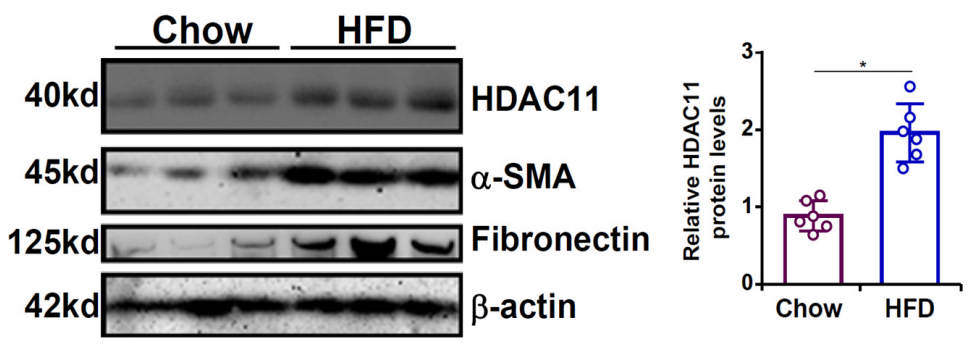

F

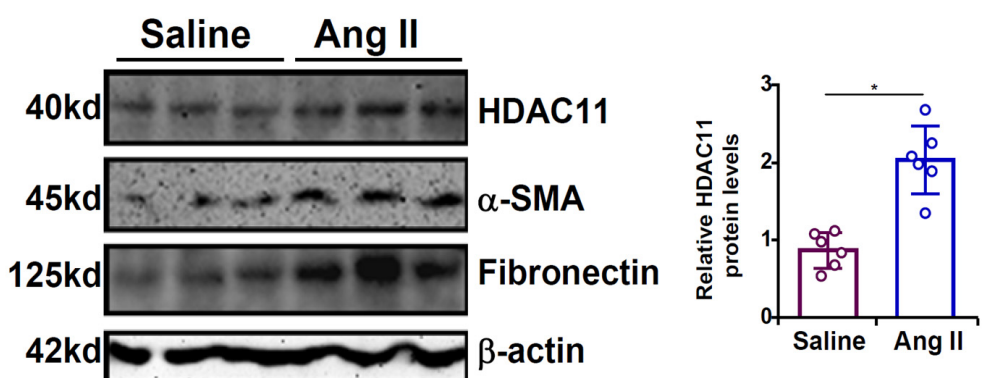

H
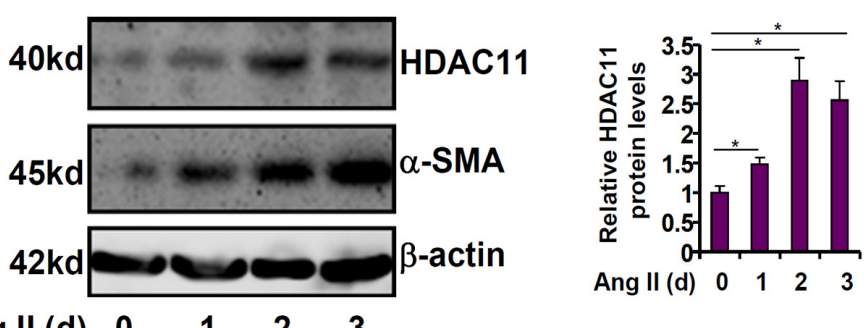

$\begin{array}{lllll}\text { Ang II (d) } & 0 & 1 & 2 & 3\end{array}$

FIGURE 1 | HDAC11 is up-regulated by pro-fibrogenic stimuli in vivo and in vitro. (A,B) C57/BL6 mice were subjected to the UUO procedure or the sham procedure. The mice were sacrificed 2 weeks after the surgery and renal HDAC11 expression was examined by qPCR and Western blot analysis. $N=6$ mice for each group. (C,D) C57/BL6 mice were fed a high-fat diet (HFD) or a control diet for 16 weeks. Renal HDAC11 expression was examined by qPCR and Western blot analysis. N = 6 mice for each group. (E,F) C57/BL6 mice were implanted with an Ang II minipump as described in section "Materials and Methods." Renal HDAC11 expression was examined by qPCR and Western blot analysis. $N=6$ mice for each group. (G,H) HK-2 cells were treated with or without Ang II (1 $\mu$ M) and harvested at indicated time points. HDAC11 expression was examined by qPCR and Western blot analysis. 
A

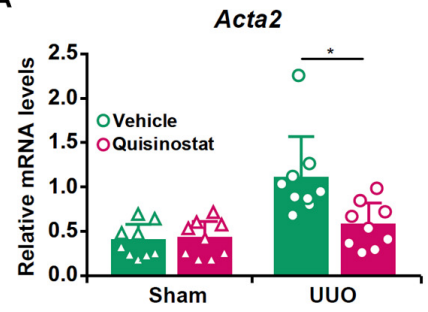

D
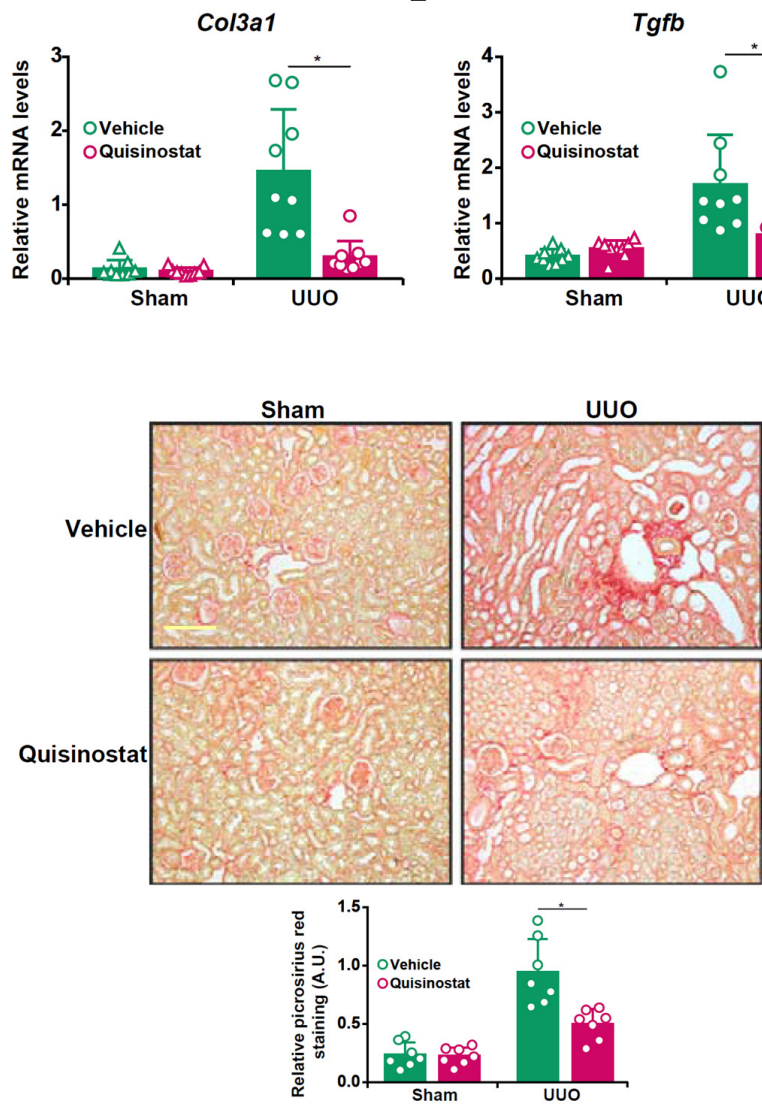

H

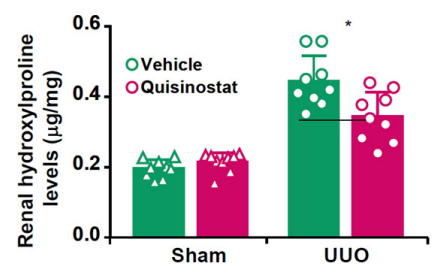

B

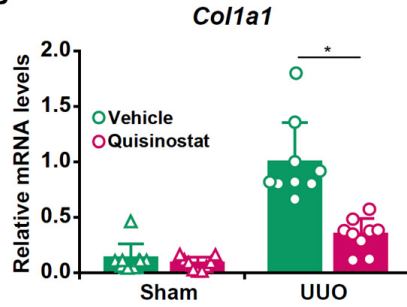

E

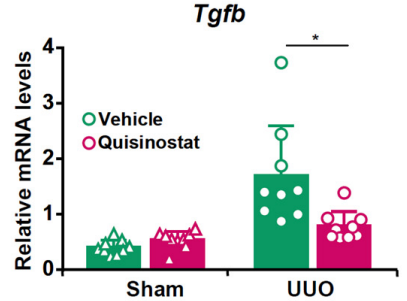

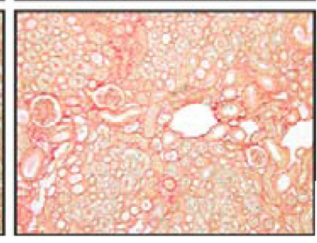

C

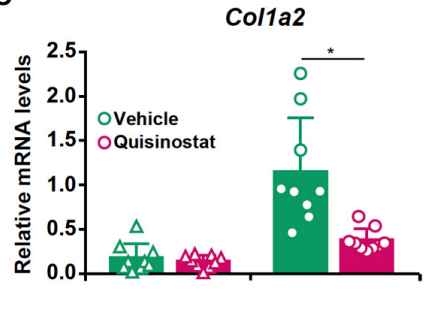

G
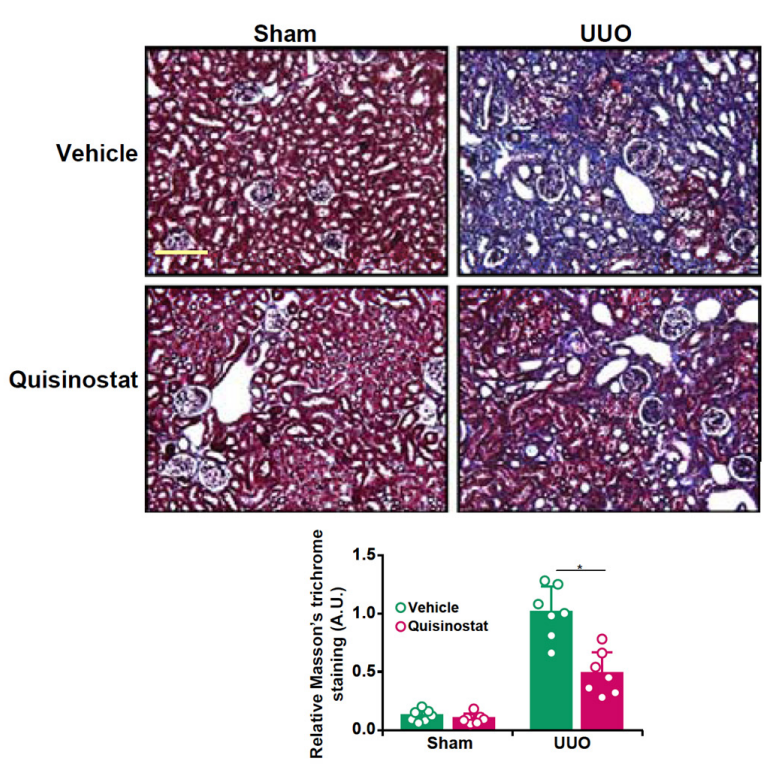

J
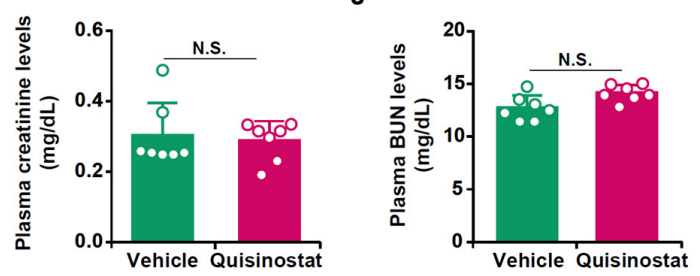

FIGURE 2 | Inhibition of HDAC11 by quisinostat attenuates renal fibrosis in mice. Renal fibrosis was induced in C57/BL6 mice by UUO. After the surgery, the mice were injected with quisinostat or vehicle as described in section "Materials and Methods." (A-E) Expression levels of Col1a1 (A), Col1a1 (B), Col3a1 (C), Acta2 (D), and Tgfb (E) were examined by qPCR. (F,G) Paraffin sections were stained with picrosirius red and Masson's trichrome. (H) Hydroxylproline levels. (I) Plasma BUN levels. (J) Plasma creatinine levels. $N=7 \sim 9$ mice for each group. 
A

\section{HDAC11}

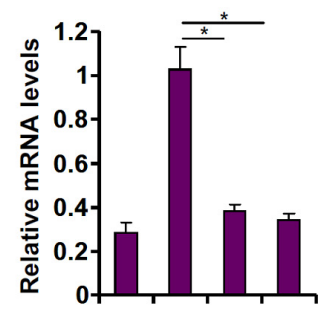

Ang II -+++

$\mathrm{SCR}++-$

SCR ++- siHDAC11\#1 $-2-1-$ siHDAC11\#1 $-2+-1+$

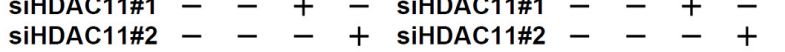

B
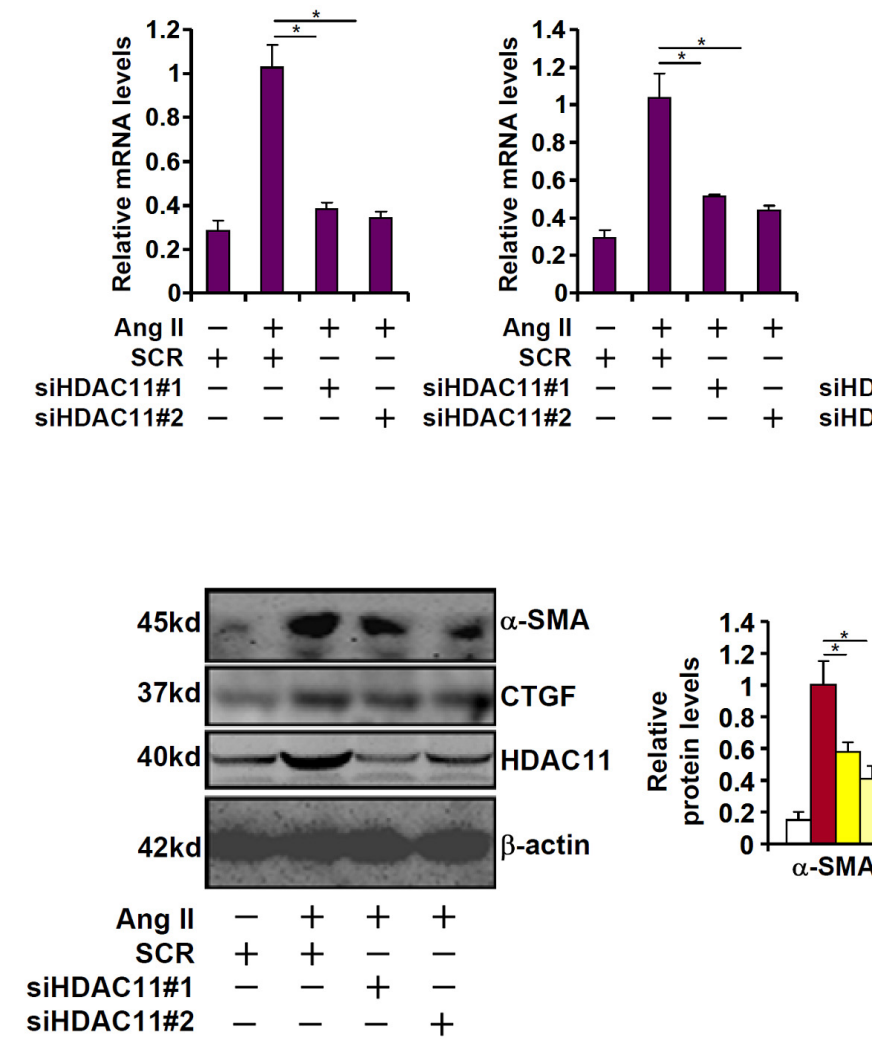

ACTA2

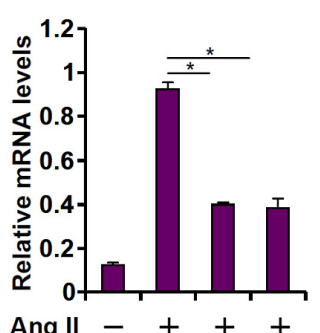

Ang II -+++

SCR ++--

$\begin{array}{llll}\text { SiHDAC11\#1 } & - & - & + \\ \text { siHDAC11\#2 } & - & - & -\end{array}$

-+ SIHDAC11\#2 -
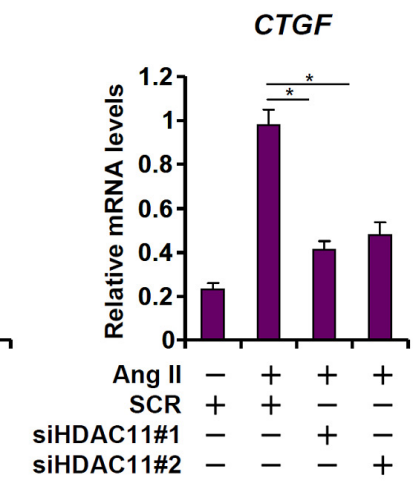

C

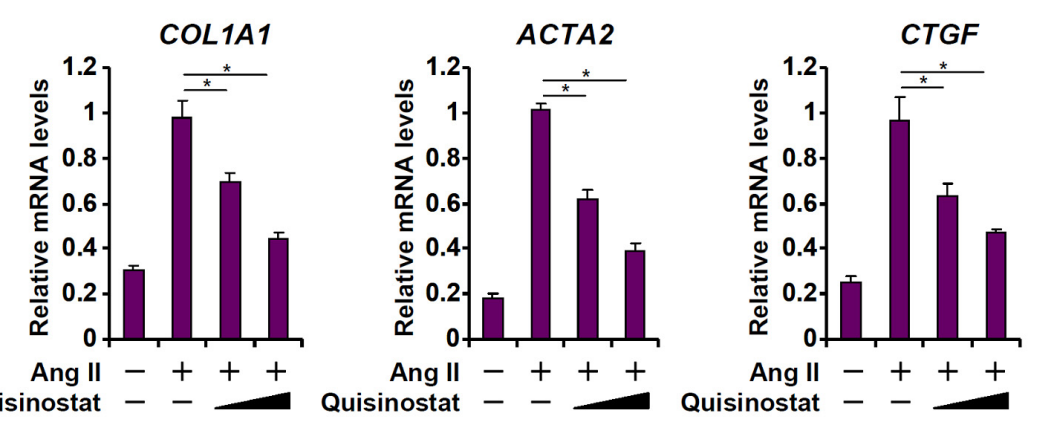

D

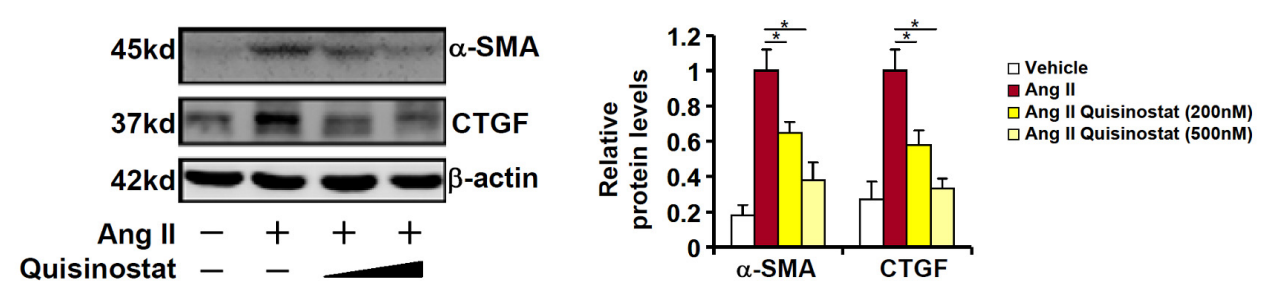

FIGURE 3 | HDAC11 mediates Ang II induced pro-fibrogenic response in HK-2 cells. (A,B) HK-2 cells were transfected with small interfering RNAs targeting HDAC11 or scrambled siRNA (SCR) followed by treatment with Ang II (1 $\mu \mathrm{M})$. Expression levels were examined by qPCR and Western blot analysis. (C,D) HK-2 cells were treated with Ang II (1 $\mu \mathrm{M})$ in the presence or absence of quisinostat (200 nM, $500 \mathrm{nM})$. Expression levels were examined by qPCR and Western blot analysis. 
A

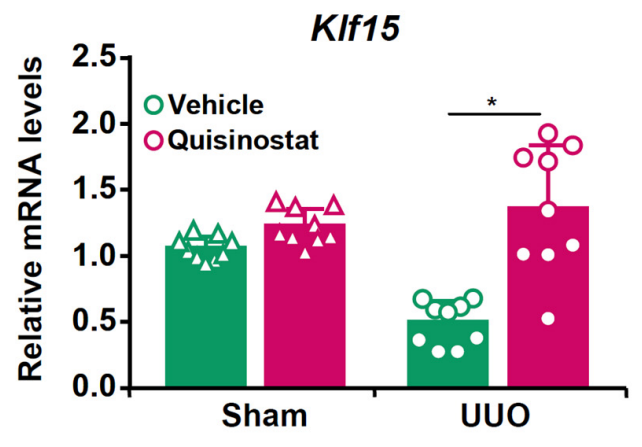

B

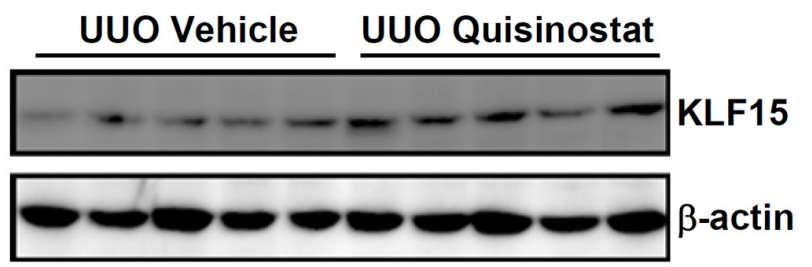

C

D
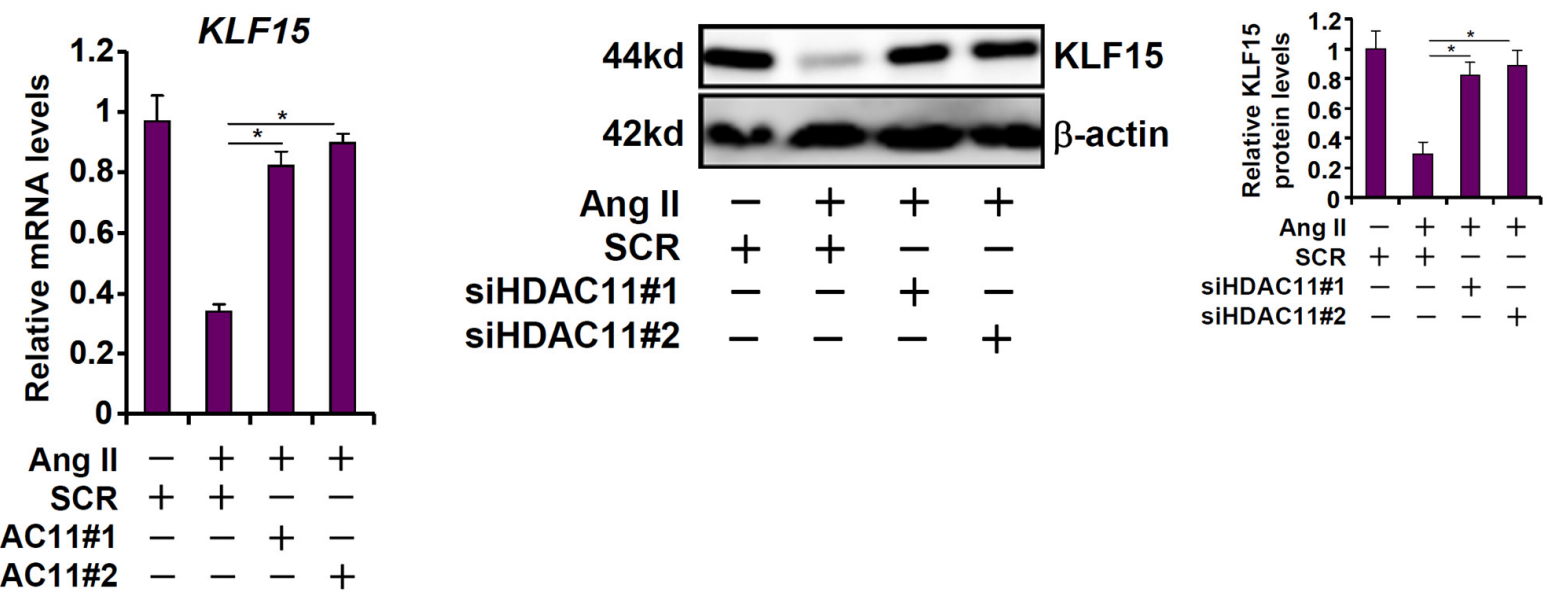

$\mathbf{E}$

$\mathbf{F}$
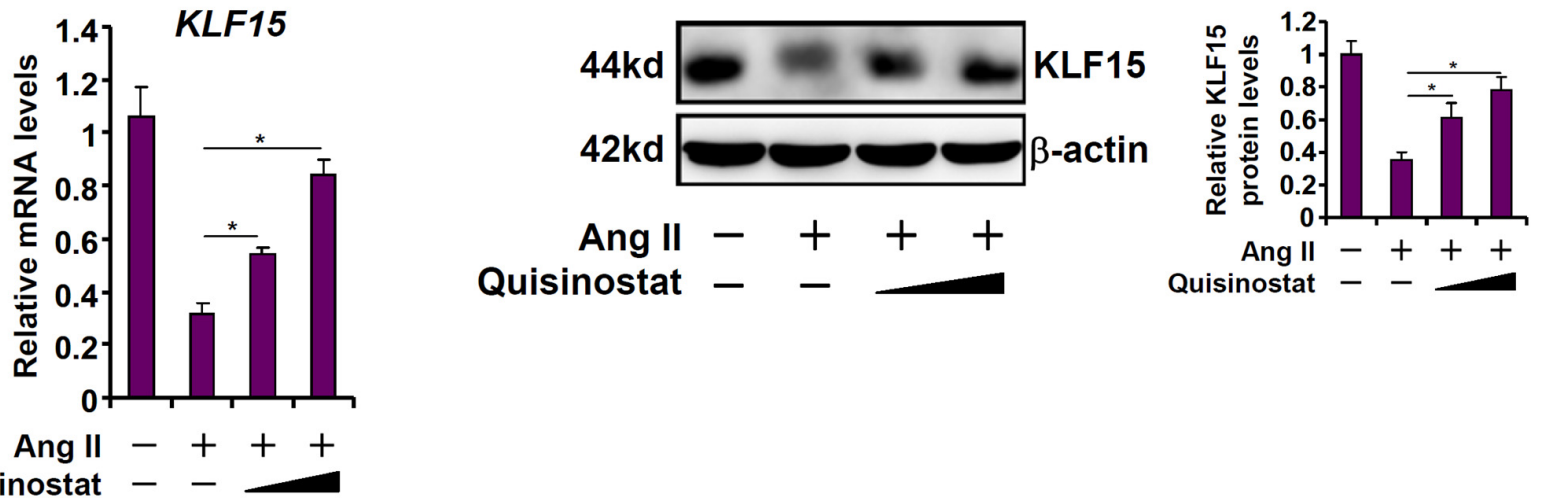

Ang II -+++ Quisinostat - -

FIGURE 4 | HDAC11 is essential for Ang II induced KLF15 repression. (A,B) Renal fibrosis was induced in C57/BL6 mice by UUO. After the surgery, the mice were injected with quisinostat or vehicle as described in section "Materials and Methods." Renal KLF15 expression was examined by qPCR and Western blot analysis. (C,D) HK-2 cells were transfected with small interfering RNAs targeting HDAC11 or scrambled sIRNA (SCR) followed by treatment with Ang II (1 $\mu$ M). KLF15 expression was examined by QPCR and Western blot analysis. (E,F) HK-2 cells were treated with Ang II $(1 \mu \mathrm{M})$ in the presence or absence of quisinostat $(200 \mathrm{nM}$, $500 \mathrm{nM})$. KLF15 expression was examined by qPCR and Western blot analysis. 
A
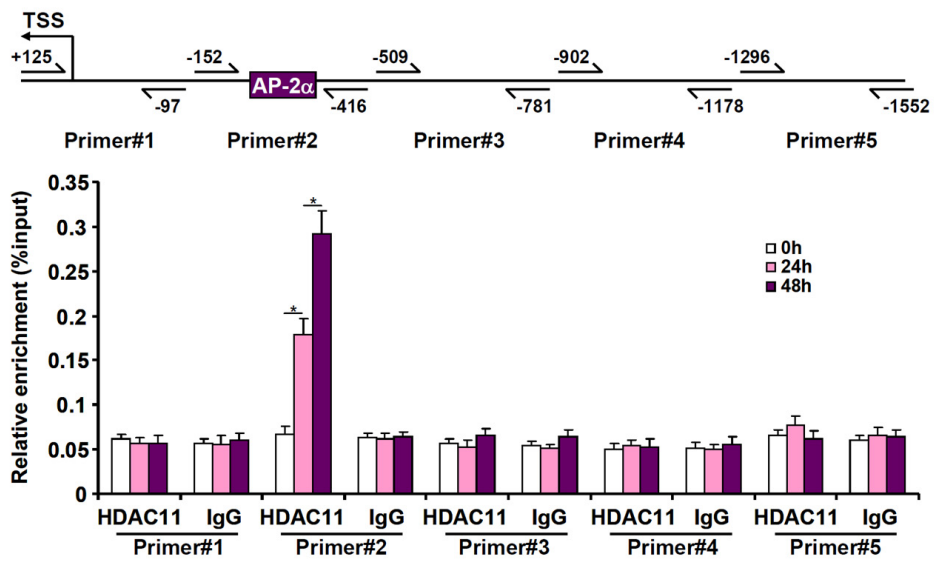

B

C
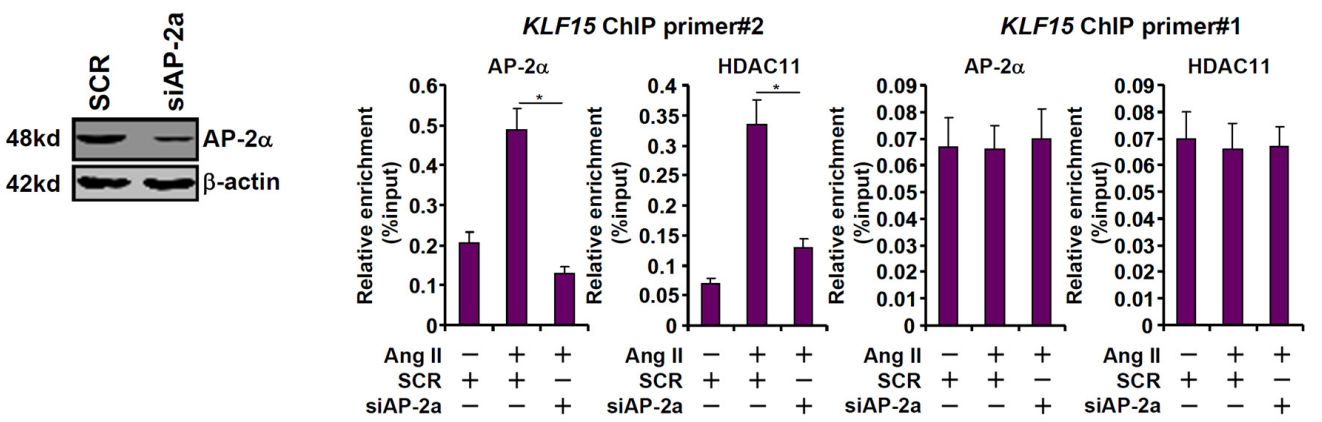

D

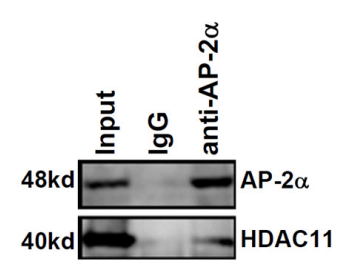

E

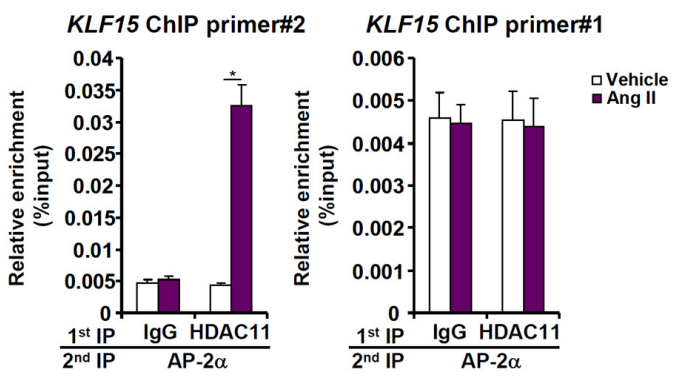

F

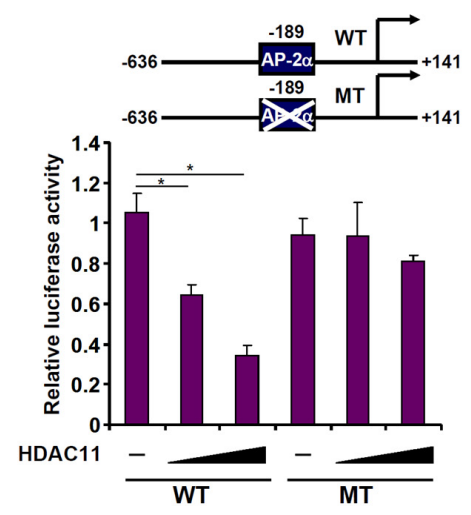

FIGURE 5 | HDAC11 interacts with AP-2 $\alpha$ to repress KLF15 transcription. (A) HK-2 cells were treated with Ang II (1 $\mu$ M). The cells were harvested at indicated time points and ChIP assays were performed with an anti-HDAC11 antibody or lgG. (B,C) HK-2 cells were transfected with siRNA targeting AP-2 $\alpha$ or SCR followed by (Continued) 
FIGURE 5 | Continued

treatment with Ang II (1 $\mu \mathrm{M})$ for $48 \mathrm{~h}$. ChIP assays were performed with an anti-HDAC11 antibody. (D) HK-2 cells were treated with Ang II (1 $\mu$ M) for $48 \mathrm{~h}$. Nuclear proteins were extracted and immunoprecipitation was performed with indicated antibodies. (E) HK-2 cells were treated with or without Ang $I$ ( $1 \mu \mathrm{M})$ for $48 \mathrm{~h}$. Re-ChIP assay was performed with indicated antibodies. (F) Wild type or mutant KLF15 promoter-luciferase construct was transfected into HK-2 cells with or without HDAC11. Luciferase activities were normalized by GFP fluorescence and protein concentration.

an Ang II-infusion minipump was implanted subcutaneously in C57/BL6 mice for 4 weeks to induce renal fibrosis. Again, it was observed that HDAC11 expression levels were augmented in the Ang II-infused fibrotic kidneys as opposed to the saline-infused kidneys (Figures 1E,F). We also determined whether HDAC11 up-regulation in the fibrotic kidneys occurred universally or only in specific cell compartments. To this end, primary renal tubular epithelial cells, podocytes, and fibroblasts were isolated from the UUO mice or the sham mice. Of interest, HDAC11 was only up-regulated in the tubular epithelial cells isolated from the UUO mice compared to the sham mice but not the podocytes or the fibroblast cells (Supplementary Figure S1). Therefore we focused on the tubular epithelial cells to investigate the role of HDAC11 hereafter.

Angiotensin II (Ang II) has been reported to play a key role promoting renal fibrosis in model animals (Chevalier, 2006; Pandey et al., 2016; Tikoo et al., 2016; Xu et al., 2017). Next, cultured human renal tubular epithelial cells (HK-2) were treated with Ang II. HDAC11 was gradually up-regulated by Ang II stimulation with a similar kinetics as $\alpha$-SMA: there was a small increase in HDAC11 expression $24 \mathrm{~h}$ after the addition of Ang II; HDAC11 expression continued to rise at $48 \mathrm{~h}$ and declined slightly at $72 \mathrm{~h}$ (Figures 1G,H). In order to determine whether Ang II could directly stimulate HDAC11 transcription, a human HDAC11 promoter-luciferase construct was transfected into HK2 cells. Ang II treatment significantly up-regulated the HDAC11 promoter activity (Supplementary Figure S2); notably, mutation of a conserved NF- $\kappa \mathrm{B}$ site site within the HDAC11 promoter abrogated induction by Ang II indicating that NF- $\mathrm{B}$ could potentially mediate the effect of Ang II treatment on HDAC11 transcription. Combined, these data suggest that there might be a positive correlation between HDAC11 and renal fibrosis both in vivo and in vitro.

\section{Inhibition of HDAC11 by Quisinostat Attenuates Renal Fibrosis in Mice}

Based on the observation that HDAC11 was up-regulated in the fibrotic kidneys, we sought to evaluate the effect of pharmaceutical inhibition of HDAC11 on renal fibrosis in the UUO model. To this end, a small-molecule HDAC11 inhibitor quisinostat (Zhou et al., 2017) was administered via peritoneal injection twice a week after the UUO procedure. Quantitative PCR showed that HDAC11 inhibition by quisinostat suppressed the induction of pro-fibrogenic genes such as $\alpha$ SMA (Acta2, Figure 2A), collagen type I (Col1a1/Colla2, Figures 2B,C), collagen type III (Colla3, Figure 2D), and transforming growth factor ( $T g f b$, Figure 2E) in the kidneys. Picrosirius red staining (Figure 2F) and Masson's trichrome staining (Figure 2G) confirmed that renal fibrosis was less extensive in the mice injected with quisinostat than the mice injected with vehicle. Quantification of hydroxylproline levels, as a measurement of total collagen synthesis, in the kidneys showed that administration of quisinostat attenuated renal fibrosis (Figure 2H). Of note, HDAC11 inhibition did not alter UUO-induced impairment of glomerular filtration as evidenced by comparable plasma BUN levels (Figure 2I) and creatinine levels (Figure 2J). We also observed that renal inflammation, as measured by infiltration of $\mathrm{CD}^{+}$lymphocytes and $\mathrm{CD} 45^{+}$ leukocytes as well as expression levels of pro-inflammatory mediators in the kidneys, was significantly dampened by quisinostat administration (Supplementary Figure S3).

\section{HDAC11 Mediates Ang II Induced Pro-fibrogenic Response in HK-2 Cells}

We next examined the effect of HDAC11 deletion or inhibition on Ang II induced pro-fibrogenic response in HK-2 cells. Exposure of HK-2 cells markedly stimulated the expression of collagen type I, $\alpha$-SMA, and CTGF as expected; HDAC11 knockdown by two separate pairs of siRNAs abrogated the induction of these pro-fibrogenic genes (Figures 3A,B). Alternatively, co-treatment with quisinostat, the HDAC11 inhibitor, suppressed Ang II-induced pro-fibrogenic response in a dose-dependent manner (Figures 3C,D).

\section{HDAC11 Is Essential for Ang II Induced KLF15 Repression}

Kruppel-like factor 15 (KLF15) is a transcription factor that has been shown to suppress UUO ( $\mathrm{Gu}$ et al., 2017a) and Ang II (Gu et al., 2017b) induced renal fibrosis in mice. Of note, KLF15 expression was down-regulated in the UUO kidneys compared to the sham kidneys, which was alleviated by quisinostat administration (Figures 4A,B). We then hypothesized that HDAC11 might mediate Ang II induced repression of KLF15 in RTECs. Ang II treatment led to a reduction of KLF15 mRNA (Figure 4C) and protein (Figure 4D) levels; HDAC11 knockdown largely normalized KLF15 expression. Similarly, HDAC11 inhibition by quisinostat dose-dependently antagonized repression of KLF15 expression by Ang II treatment (Figures 4E,F).

To determine the region within the KLF15 promoter to which HDAC11 might bind, ChIP assays were performed with an anti-HDAC11 antibody or a control IgG. Precipitated DNA was amplified by primers that span $\sim 1.5 \mathrm{~kb}$ of the proximal KLF15 promoter. As shown in Figure 5A, Ang II treatment specifically stimulated the recruitment of HDAC11 to a region within the proximal KLF15 promoter $(-152 /-416)$ that contains a binding site for the transcriptional repressor AP- $2 \alpha$. Small interfering RNA targeting AP-2a was used to deplete endogenous AP- $2 \alpha$ to test the possibility that AP- $2 \alpha$ might be responsible for recruiting HDAC11 to the KLF15 promoter (Figure 5B). ChIP 
A

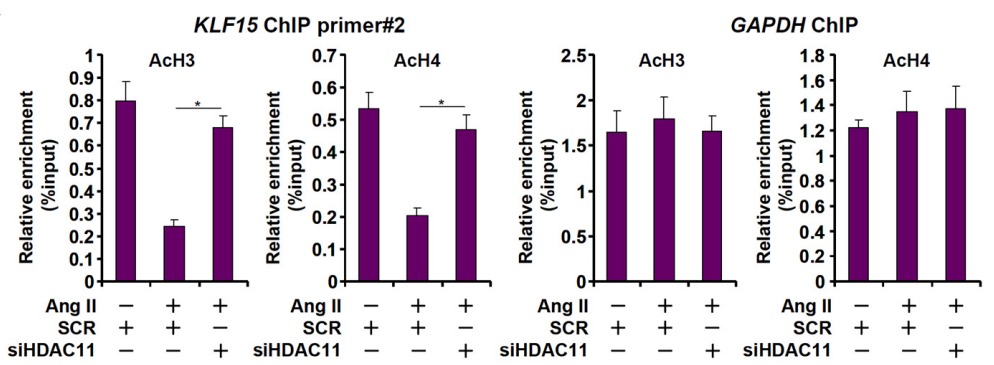

B

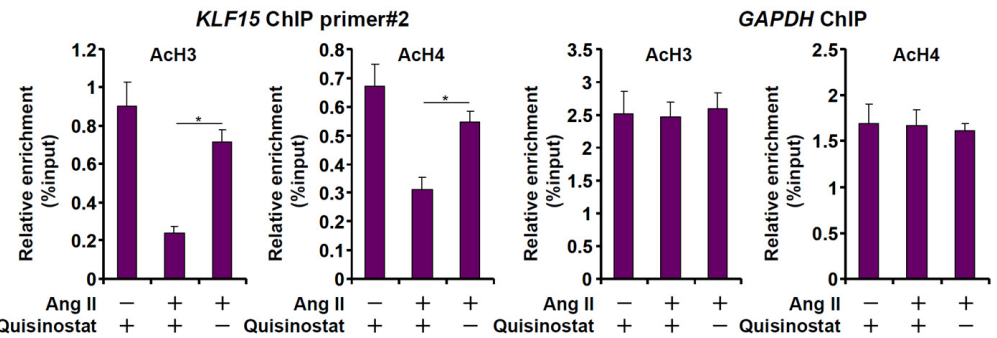

C

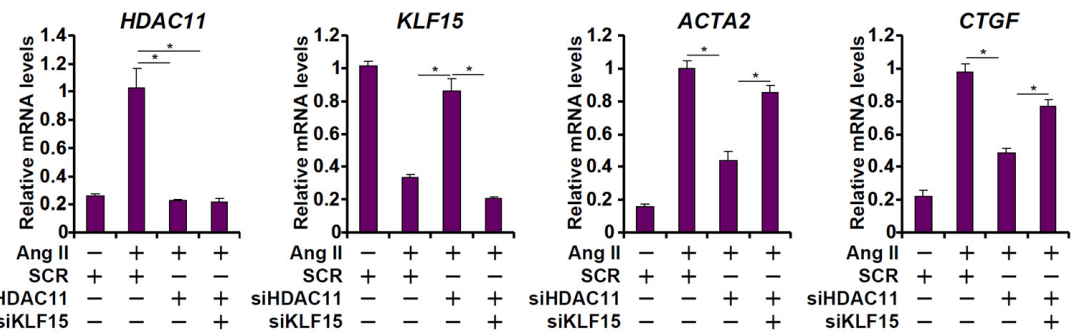

D

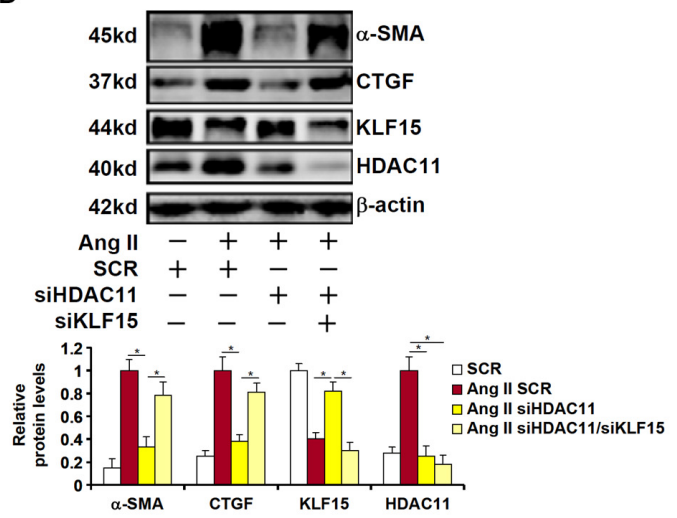

$\mathbf{F}$

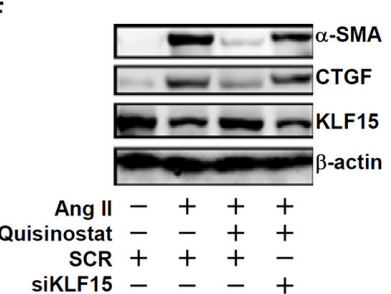

E

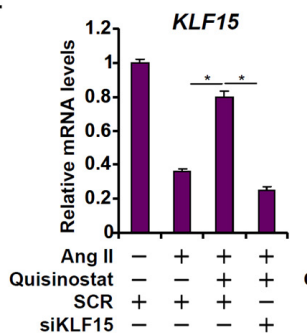

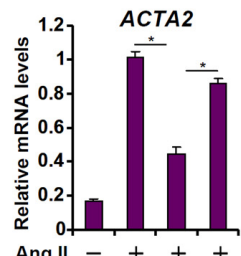

Ang II -+++

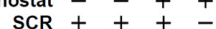

SCR +++
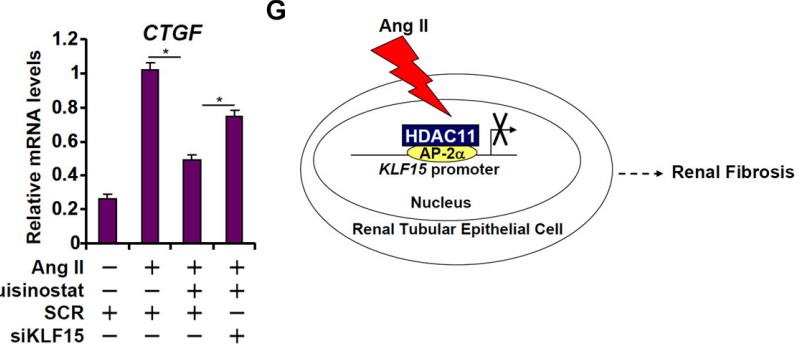

FIGURE 6 | HDAC11 promotes pro-fibrogenic response through KLF15 in HK-2 cells. (A) HK-2 cells were transfected with small interfering RNAs targeting HDAC11 or scrambled sIRNA (SCR) followed by treatment with Ang II (1 $\mu \mathrm{M})$. ChIP assays were performed with anti-acetyl H3 and anti-acetyl H4 antibodies. (B) HK-2 cells were treated with Ang II (1 $\mu \mathrm{M})$ in the presence or absence of quisinostat $(500 \mathrm{nM})$. ChIP assays were performed with anti-acetyl H3 and anti-acetyl $\mathrm{H} 4$ antibodies. 
FIGURE 6 | Continued

(C,D) HK-2 cells were transfected with small interfering RNAs targeting HDAC11, KLF15, or scrambled siRNA (SCR) followed by treatment with Ang II (1 $\mu$ M). Expression levels were examined by qPCR and Western blot analysis. (E,F) HK-2 cells were transfected with small interfering RNAs targeting KLF15, or scrambled siRNA (SCR) followed by treatment with Ang II (1 $\mu \mathrm{M})$ and/or quisinostat (500 nM). Expression levels were examined by qPCR and Western blot analysis. (G) A schematic model.

assays revealed that Ang II treatment significantly augmented the occupancies of both AP- $2 \alpha$ and HDAC11 on the KLF15 promoter; AP- $2 \alpha$ knockdown, however, abolished the binding of both (Figure 5C). Additional experiments were performed to confirm the interaction between HDAC11 and AP- $2 \alpha$. An anti-AP- $2 \alpha$ antibody simultaneously precipitated both AP- $2 \alpha$ and HDAC11 from nuclear lysates extracted from HK-2 cells, suggesting that AP- $2 \alpha$ and HDAC11 may form a complex (Figure 5D). Further, Re-ChIP assay showed that Ang II treatment strongly enhanced the interaction between AP- $2 \alpha$ and HDAC11 on the KLF15 promoter (Figure 5E). Functionally, over-expression of HDAC11 dose-dependently repressed the KLF15 promoter activity, which was blunted by the mutation of the AP- $2 \alpha$ site (Figure 5F). Together, these data seem to support a role for the AP- $2 \alpha$-HDAC11 complex in mediating Ang II induced KLF15 repression in tubular epithelial cells.

\section{HDAC11 Promotes Pro-fibrogenic Response Through KLF15 in HK-2 Cells}

Histone deacetylase 11 is an atypical (class IV) histone deacetylase. We examined the effect of HDAC1 depletion/inhibition on hisone acetylation surrounding the KLF15 promoter. When HK-2 cells were exposed to Ang II, there was a simultaneous loss of acetyl $\mathrm{H} 3$ and acetyl H4 from the KLF15 promoter, but not from the GAPDH promoter, consistent with repression of KLF15 transcription (Figures 6A,B). HDAC11 depletion by siRNA (Figure 6A) or HDAC11 inhibition by quisinostat (Figure 6B) largely restored histone acetylation surrounding the KLF15 promoter, indicating that HDAC11 likely contributes to KLF15 repression by modulating histone acetylation levels. We then asked whether the ability of HDAC11 to promote Ang II induced pro-fibrogenic response relies on KLF15. As shown in Figures 6C,D, whereas HDAC11 knockdown suppressed induction of pro-fibrogenic gene expression by Ang II, simultaneous depletion of HDAC11 and KLF15 restored the pro-fibrogenic response induced by Ang II. Similarly, the loss of KLF15 antagonized the effect of quisinostat and enabled Ang II to promote fibrogenesis in HK-2 cells (Figures 6E,F). We therefore conclude that KLF15 may be the primary target of HDAC11 during Ang II induced pro-fibrogenic response in tubular epithelial cells.

\section{DISCUSSION}

Epigenetic regulation of renal fibrosis is one of the most actively investigated areas in deciphering the pathogenesis of and finding the solutions for end-stage renal diseases (Tampe and Zeisberg, 2014; Kato and Natarajan, 2019). Here we report that the histone deacetylase HDAC11 promotes renal fibrosis by epigenetically repressing the transcription of KLF15, an anti-fibrogenic factor. Inhibition of HDAC11 with a smallmolecule compound quisinostat attenuates UUO-induced renal fibrosis in mice. Therefore, our data provide the proof-ofconcept for targeting HDAC11 as a potential therapeutic solution against renal fibrosis. However, there are a few caveats that deserve further attention regarding the current working model (Figure 6G). First, we focused our investigation on renal tubular epithelial cells. It has been reported that other cell types, including fibroblasts, endothelial cells, and myeloid cells, can contribute to renal fibrosis (Zeisberg and Neilson, 2010; LeBleu et al., 2013; Mack and Yanagita, 2015); relatively little is known regarding the role of HDAC11 in these cells. HDAC11 plays a regulatory role in myeloid cells (Yanginlar and Logie, 2018). For instance, Wang et al. (2011) have previously reported that HDAC11 represses the transcription of IL-10 in macrophages. Administration of IL-10, coincidently, can suppress renal fibrosis in rats subjected to 5/6 nephrectomy ( $\mathrm{Mu}$ et al., 2005). In addition, HDAC11 can modulate the function of myeloid derived suppressor cells (MDSCs), a heterogeneous population of immune cells specialized in the suppression of $\mathrm{T}$ lymphocyte function (Sahakian et al., 2015). Hsieh et al. (2018) have shown that administration of MDSCs ameliorates renal fibrosis in diabetic mice. These observations collectively appear to suggest that HDAC11 may contribute to renal fibrosis by regulating the immune microenvironment in the kidneys. Second, HDACs, in addition to removing the acetyl group from histones, can also de-acetylate non-histone proteins. The class III HDAC SIRT1 mitigates renal fibrosis, in part, by deacetylating and deactivating SMAD3, a key transcription factor involved in fibrogenesis ( $\mathrm{Li}$ et al., 2010). HDAC1 and HDAC2 can promote the deacetylation of STAT1, which prevents its binding to and inhibition of NF$\kappa \mathrm{B}$ allowing the latter to stimulate a pro-fibrogenic transcription program in mesangial cells (Kumar et al., 2017). The acetylation status and thus activity of STAT3, a pro-fibrogenic transcription factor, can also be modulated by HDACs during renal fibrosis (Ni et al., 2014). Since specific non-histone substrates for HDAC11 have yet to be identified, profiling the HDAC11 interactome in the kidneys may provide novel insight into its mode of action in the context of renal fibrosis.

There is growing body of evidence that supports an antifibrotic role of KLF15 in the kidneys. Mei and colleagues were among the first to report that KLF15 levels were decreased in the kidneys of rats in a model of chronic renal disease (CKD) and that KLF15 deletion sensitized the mice to the development of renal fibrosis (Gao et al., 2011; $\mathrm{Gu}$ et al., 2017a). Mechanistically, KLF15 may regulate renal fibrosis by suppressing the ERK/MAPK, the JNK/MAPK, and the Wnt/ $\beta$-catenin pathways (Gao et al., 2013; Gu et al., 2017a). More recently, $\mathrm{Lu}$ et al. have shown that KLF15 
may regulate renal fibrosis by modulating activity of the TGF- $\beta$ downstream mediators SMAD2/3 via its trans-activation domain (TAD) (Lu et al., 2019). Although KLF15 levels can be downregulated by a host of pro-fibrotic stimuli in the kidneys the underlying mechanism remains obscure. We show here that AP$2 \alpha$ recruits HDAC11 to repress KLF15 transcription in tubular epithelial cells. Although a vast majority of the studies conducted so far have portrayed AP- $2 \alpha$ as a regulator of lineage specification during embryogenesis and cancer development and progression in adults (Eckert et al., 2005; Kolat et al., 2019), there is indication that AP- $2 \alpha$ may play a key role in cellular fibrogenic response. AP- $2 \alpha$ can promote epithelial-mesenchymal transition (EMT), a process critical to tissue fibrosis, by up-regulating TGF- $\beta$ expression (Zhang et al., 2017) and by forming a complex with ZEB1/2, the E-box binding transcriptional regulators of EMT (Dimitrova et al., 2017). Ross et al. (2019) have recently reported that deletion of TFAP2A (the gene encoding AP- $2 \alpha$ ) significantly attenuates the TGF- $\beta$ induced maturation of myofibroblasts although the underlying mechanism is unclear. Traditionally, transcription factors are notorious to target in drug development. Recent successes in "drugging" such transcription factors as p53 (Khoo et al., 2014) and c-Myc (Dang et al., 2017) may shed some light on this issue should further evidence present AP- $2 \alpha$ and/or KLF15 as a desirable target in the intervention of renal fibrosis.

There are several limitations of the present study that necessitate cautious interpretation of the data within. First, we relied exclusively on quisinostat to evaluate the effect of HDAC11 on renal fibrosis in vivo. Quisinostat is not a strictly specific HDAC11 inhibitor because it can, with equivalent potency, target several other HDACs that have been demonstrated to play regulatory roles in renal fibrosis (Arts et al., 2009). Therefore, it remains uncertain whether the anti-fibrotic effects of quisinostat administration are achieved by HDAC11 inhibition. Future studies employing tissue-specific HDAC11 knockout mice (Bagchi et al., 2018) will hopefully provide solid genetic evidence to ascertain the role of HDAC11 in renal fibrosis. Second, the in vitro data were based on a single cell model (Ang II treated human tubular epithelial cells), which makes it difficult to reconcile them with the in vivo data especially in the light of the recent finding that tubular epithelial cells derived myofibroblasts only constitute a small fraction of the overall population of myofibroblasts in the fibrotic kidneys in mice (LeBleu et al., 2013). Therefore the issue as to whether

\section{REFERENCES}

Arts, J., King, P., Marien, A., Floren, W., Belien, A., Janssen, L., et al. (2009). JNJ-26481585, a novel "second-generation" oral histone deacetylase inhibitor, shows broad-spectrum preclinical antitumoral activity. Clin. Cancer Res. 15, 6841-6851. doi: 10.1158/1078-0432.ccr-09-0547

Bagchi, R. A., Ferguson, B. S., Stratton, M. S., Hu, T., Cavasin, M. A., Sun, L., et al. (2018). HDAC11 suppresses the thermogenic program of adipose tissue via BRD2. JCI Insight 3:e120159.

Chevalier, R. L. (2006). Obstructive nephropathy: towards biomarker discovery and gene therapy. Nat. Clin. Pract. Nephrol. 2, 157-168. doi: 10.1038/ncpneph0098

Choi, S. Y., Kee, H. J., Kurz, T., Hansen, F. K., Ryu, Y., Kim, G. R., et al. (2016). Class I HDACs specifically regulate E-cadherin expression in human renal epithelial cells. J. Cell. Mol. Med. 20, 2289-2298. doi: 10.1111/jcmm.12919
HDAC11-driven synthesis of pro-fibrogenic molecules in tubular epithelial cells in response to Ang II treatment plays a significant role in the pathogenesis of renal fibrosis in vivo needs to be revisited in the future.

In summary, our data suggest that an AP-2 $\alpha$-HDAC11-KLF15 axis is involved in the pathogenesis of renal fibrosis. Smallmolecule inhibitors that target this axis may be considered as a potential therapeutic strategy for the treatment of endstage renal diseases.

\section{DATA AVAILABILITY STATEMENT}

The datasets generated for this study are available on request to the corresponding author.

\section{ETHICS STATEMENT}

The animal study was reviewed and approved by the Nanjing Medical University Committee on Ethical Conduct of Animal Experiments.

\section{AUTHOR CONTRIBUTIONS}

YX conceived the project and wrote the manuscript with inputs from all authors. LM and XW designed the experiments. LM, LL, TZ, HQ, and XW performed the experiments and collected and analyzed the data. TZ and XW provided funding and supervision.

\section{FUNDING}

This work was supported, in part, by grants from the National Natural Science Foundation of China (81670618 and 81970638).

\section{SUPPLEMENTARY MATERIAL}

The Supplementary Material for this article can be found online at: https://www.frontiersin.org/articles/10.3389/fcell.2020.00235/ full\#supplementary-material

Coresh, J. (2017). Update on the burden of CKD. J. Am. Soc. Nephrol. 28, 1020-1022. doi: 10.1681/asn.2016121374

Dang, C. V., Reddy, E. P., Shokat, K. M., and Soucek, L. (2017). Drugging the 'undruggable' cancer targets. Nat. Rev. Cancer 17, 502-508. doi: 10.1038/nrc. 2017.36

Dimitrova, Y., Gruber, A. J., Mittal, N., Ghosh, S., Dimitriades, B., Mathow, D., et al. (2017). TFAP2A is a component of the ZEB1/2 network that regulates TGFB1-induced epithelial to mesenchymal transition. Biol. Direct. $12: 8$.

Eckert, D., Buhl, S., Weber, S., Jager, R., and Schorle, H. (2005). The AP-2 family of transcription factors. Genome Biol. 6:246.

Edeling, M., Ragi, G., Huang, S., Pavenstadt, H., and Susztak, K. (2016). Developmental signalling pathways in renal fibrosis: the roles of Notch, Wnt and Hedgehog. Nat. Rev. Nephrol. 12, 426-439. doi: 10.1038/nrneph.2016.54 
Falke, L. L., Gholizadeh, S., Goldschmeding, R., Kok, R. J., and Nguyen, T. Q. (2015). Diverse origins of the myofibroblast-implications for kidney fibrosis. Nat. Rev. Nephrol. 11, 233-244. doi: 10.1038/nrneph.2014.246

Fan, Z., Li, N., Xu, Z., Wu, J., Fan, X., and Xu, Y. (2019). An interaction between MKL1, BRG1, and C/EBPbeta mediates palmitate induced CRP transcription in hepatocytes. Biochim. Biophys. Acta Gene Regul. Mech. 1862:194412. doi: 10.1016/j.bbagrm.2019.194412

Fan, Z., Li, Z., Yang, Y., Liu, S., Guo, J., and Xu, Y. (2017). HIF-1alpha coordinates epigenetic activation of SIAH1 in hepatocytes in response to nutritional stress. Biochim. Biophys. Acta Gene Regul. Mech. 1860, 1037-1046. doi: 10.1016/j. bbagrm.2017.08.002

Gao, X., Huang, L., Grosjean, F., Esposito, V., Wu, J., Fu, L., et al. (2011). Lowprotein diet supplemented with ketoacids reduces the severity of renal disease in 5/6 nephrectomized rats: a role for KLF15. Kidney Intern. 79, 987-996. doi: 10.1038/ki.2010.539

Gao, X., Wu, G., Gu, X., Fu, L., and Mei, C. (2013). Kruppel-like factor 15 modulates renal interstitial fibrosis by ERK/MAPK and JNK/MAPK pathways regulation. Kidney Blood Pressure Res. 37, 631-640. doi: 10.1159/000355743

Gewin, L. S. (2018). Renal fibrosis: Primacy of the proximal tubule. Matrix Biol. 6, 248-262. doi: 10.1016/j.matbio.2018.02.006

Gu, X., Mallipattu, S. K., Guo, Y., Revelo, M. P., Pace, J., Miller, T., et al. (2017a). The loss of Kruppel-like factor 15 in Foxd1(+) stromal cells exacerbates kidney fibrosis. Kidney Intern. 92, 1178-1193. doi: 10.1016/j.kint.2017. 03.037

Gu, X., Xu, D., Fu, L., Wang, Y., Mei, C., and Gao, X. (2017b). KLF 15 works as an early anti-fibrotic transcriptional regulator in Ang II-induced renal fibrosis via down-regulation of CTGF expression. Kidney Blood Pressure Res. 42, 999-1012. doi: 10.1159/000485349

Hsieh, C. C., Lin, C. L., He, J. T., Chiang, M., Wang, Y., Tsai, Y. C., et al. (2018). Administration of cytokine-induced myeloid-derived suppressor cells ameliorates renal fibrosis in diabetic mice. Stem Cell Res. Ther. 9:183.

Humphreys, B. D., Lin, S. L., Kobayashi, A., Hudson, T. E., Nowlin, B. T., Bonventre, J. V., et al. (2010). Fate tracing reveals the pericyte and not epithelial origin of myofibroblasts in kidney fibrosis. Am. J. Pathol. 176, 85-97. doi: 10.2353/ajpath.2010.090517

Jenuwein, T., and Allis, C. D. (2001). Translating the histone code. Science 293, 1074-1080. doi: 10.1126/science. 1063127

Kato, M., and Natarajan, R. (2019). Epigenetics and epigenomics in diabetic kidney disease and metabolic memory. Nat. Rev. Nephrol. 15, 327-345. doi: 10.1038/ s41581-019-0135-6

Khoo, K. H., Verma, C. S., and Lane, D. P. (2014). Drugging the p53 pathway: understanding the route to clinical efficacy. Nat. Rev. Drug Discov. 13, 217-236. doi: $10.1038 / \mathrm{nrd} 4236$

Kolat, D., Kaluzinska, Z., Bednarek, A. K., and Pluciennik, E. (2019). The biological characteristics of transcription factors AP-2alpha and AP-2gamma and their importance in various types of cancers. Biosci. Rep. 39:BSR20181928.

Kong, M., Chen, X., Lv, F., Ren, H., Fan, Z., Qin, H., et al. (2019a). Serum response factor (SRF) promotes ROS generation and hepatic stellate cell activation by epigenetically stimulating NCF1/2 transcription. Redox Biol. 26:101302. doi: 10.1016/j.redox.2019.101302

Kong, M., Hong, W., Shao, Y., Lv, F., Fan, Z., Li, P., et al. (2019b). Ablation of serum response factor in hepatic stellate cells attenuates liver fibrosis. J. Mol. Med. 97, 1521-1533. doi: 10.1007/s00109-019-01831-8

Kumar, P., Gogulamudi, V. R., Periasamy, R., Raghavaraju, G., Subramanian, U., and Pandey, K. N. (2017). Inhibition of HDAC enhances STAT acetylation, blocks NF-kappaB, and suppresses the renal inflammation and fibrosis in Npr1 haplotype male mice. Am. J. Physiol. Renal Physiol. 313, F781-F795.

Kunzel, S. R., Schaeffer, C., Sekeres, K., Mehnert, C. S., Schacht Wall, S. M., Newe, M., et al. (2019). Ultrasonic-augmented primary adult fibroblast isolation. J. Visual. Exp. 149. [Epub ahead print].

LeBleu, V. S., Taduri, G., O'Connell, J., Teng, Y., Cooke, V. G., Woda, C., et al. (2013). Origin and function of myofibroblasts in kidney fibrosis. Nat. Med. 19, 1047-1053.

Li, J., Qu, X., Ricardo, S. D., Bertram, J. F., and Nikolic-Paterson, D. J. (2010). Resveratrol inhibits renal fibrosis in the obstructed kidney: potential role in deacetylation of Smad3. Am. J. Pathol. 177, 1065-1071. doi: 10.2353/ajpath. 2010.090923
Li, M., Hong, W., Hao, C., Li, L., Xu, H., Li, P., et al. (2017). Hepatic stellate cellspecific deletion of SIRT1 exacerbates liver fibrosis in mice. Biochim. Biophys. Acta 1863, 3202-3211. doi: 10.1016/j.bbadis.2017.09.008

Li, N., Kong, M., Zeng, S., Xu, Z., Li, M., Hong, W., et al. (2018a). The chromatin remodeling protein BRG1 regulates APAP-induced liver injury by modulating CYP3A11 transcription in hepatocyte. Biochim. Biophys. Acta Mol. Basis Dis. 1864, 3487-3495. doi: 10.1016/j.bbadis.2018.08.003

Li, N., Li, M., Hong, W., Shao, J., Xu, H., Shimano, H., et al. (2018b). Brg1 regulates pro-lipogenic transcription by modulating SREBP activity in hepatocytes. Biochim. Biophys. Acta Mol. Basis Dis. 1864, 2881-2889. doi: 10.1016/j.bbadis. 2018.05.022

Li, Z., Chen, B., Dong, W., Xu, W., Song, M., Fang, M., et al. (2018c). Epigenetic activation of PERP transcription by MKL1 contributes to ROS-induced apoptosis in skeletal muscle cells. Biochim. Biophys. Acta Gene Regul. Mech. 1861, 905-915. doi: 10.1016/j.bbagrm.2018.07.011

Li, Z., Chen, B., Weng, X., Yu, L., Song, M., Fang, M., et al. (2018d). The histone methyltransferase SETD1A regulates thrombomodulin transcription in vascular endothelial cells. Biochim. Biophys. Acta Gene Regul. Mech. 1861, 752-761. doi: 10.1016/j.bbagrm.2018.06.004

Li, Z., Zhang, X., Liu, S., Zeng, S., Yu, L., Yang, G., et al. (2018e). BRG1 regulates NOX gene transcription in endothelial cells and contributes to cardiac ischemia-reperfusion injury. Biochim. Biophys. Acta Mol. Basis Dis. 1864, 34773486. doi: 10.1016/j.bbadis.2018.08.002

Li, Z., Li, P., Lu, Y., Sun, D., Zhang, X., and Xu, Y. (2019). A non-autonomous role of MKL1 in the activation of hepatic stellate cells. Biochim. Biophys. Acta Gene Regul. Mech. 1862, 609-618. doi: 10.1016/j.bbagrm.2019.03.001

$\mathrm{Li}, \mathrm{Z}$., and $\mathrm{Xu}, \mathrm{Y}$. (2019). Response by $\mathrm{Li}$ and $\mathrm{Xu}$ to letter regarding article, megakaryocytic leukemia 1 bridges epigenetic activation of NADPH oxidase in macrophages to cardiac ischemia-reperfusion injury. Circulation 139, e965e966.

Liu, L., Hong, W., Li, M., Ren, H., Wang, J., Xu, H., et al. (2019a). A cross talk between BRG1 and males absent on the first contributes to reactive oxygen species production in a mouse model of nonalcoholic steatohepatitis. Antioxid. Redox Signal. 30, 1539-1552. doi: 10.1089/ars.2016.6822

Liu, L., Mao, L., Wu, X., Wu, T., Liu, W., Yang, Y., et al. (2019b). BRG1 regulates endothelial-derived IL-33 to promote ischemia-reperfusion induced renal injury and fibrosis in mice. Biochim. Biophys. Acta Mol. Basis Dis. 1865, 2551-2561. doi: 10.1016/j.bbadis.2019.06.015

Liu, L., Wu, X., Xu, H., Yu, L., Zhang, X., Li, L., et al. (2018). Myocardinrelated transcription factor A (MRTF-A) contributes to acute kidney injury by regulating macrophage ROS production. Biochim. Biophys. Acta Mol. Basis Dis. 1864, 3109-3121. doi: 10.1016/j.bbadis.2018.05.026

Liu, N., He, S., Ma, L., Ponnusamy, M., Tang, J., Tolbert, E., et al. (2013). Blocking the class I histone deacetylase ameliorates renal fibrosis and inhibits renal fibroblast activation via modulating TGF-beta and EGFR signaling. PLoS One 8:e54001. doi: 10.1371/journal.pone.0054001

Liu, Z. H. (2013). Nephrology in china. Nat. Rev. Nephrol. 9, 523-528. doi: 10.1038/ nrneph.2013.146

Lu, Y. Y., Li, X. D., Zhou, H. D., Shao, S., He, S., Hong, M. N., et al. (2019). Transactivation domain of Kruppel-like factor 15 negatively regulates angiotensin II-induced adventitial inflammation and fibrosis. FASEB J. 33, 6254-6268. doi: 10.1096/fj.201801809r

Mack, M., and Yanagita, M. (2015). Origin of myofibroblasts and cellular events triggering fibrosis. Kidney Intern. 87, 297-307. doi: 10.1038/ki.2014.287

Morigi, M., Perico, L., and Benigni, A. (2018). Sirtuins in renal health and disease. J. Am. Soc. Nephrol. 29, 1799-1809. doi: 10.1681/asn.2017111218

Mu, W., Ouyang, X., Agarwal, A., Zhang, L., Long, D. A., Cruz, P. E., et al. (2005). IL-10 suppresses chemokines, inflammation, and fibrosis in a model of chronic renal disease. J. Am. Soc. Nephrol. 16, 3651-3660. doi: 10.1681/asn.2005030297

Murakami, A., Oshiro, H., Kanzaki, S., Yamaguchi, A., Yamanaka, S., Furuya, M., et al. (2010). A novel method for isolating podocytes using magnetic activated cell sorting. Nephrol. Dialy. Transplant. 25, 3884-3890. doi: 10.1093/ndt/gfq323

Ni, J., Shen, Y., Wang, Z., Shao, D. C., Liu, J., Fu, L. J., et al. (2014). Inhibition of STAT3 acetylation is associated with angiotesin renal fibrosis in the obstructed kidney. Acta Pharmacol. Sin. 35, 1045-1054. doi: 10.1038/aps.2014.42

Nishitani, Y., Iwano, M., Yamaguchi, Y., Harada, K., Nakatani, K., Akai, Y., et al. (2005). Fibroblast-specific protein 1 is a specific prognostic marker for renal 
survival in patients with IgAN. Kidney Intern. 68, 1078-1085. doi: 10.1111/j. 1523-1755.2005.00500.x

Pandey, A., Goru, S. K., Kadakol, A., Malek, V., Sharma, N., and Gaikwad, A. B. (2016). H2AK119 monoubiquitination regulates Angiotensin II receptor mediated macrophage infiltration and renal fibrosis in type 2 diabetic rats. Biochimie 131, 68-76. doi: 10.1016/j.biochi.2016.09.016

Ross, G. R., Edwards, S., Warner, C., Homar, P., Downey, F. X., Emelyanova, L., et al. (2019). Deletion of transcription factor AP-2alpha gene attenuates fibroblast differentiation into myofibroblast. J. Cell. Mol. Med. 23, 6494-6498. doi: $10.1111 /$ jcmm. 14421

Sahakian, E., Powers, J. J., Chen, J., Deng, S. L., Cheng, F., Distler, A., et al. (2015). Histone deacetylase 11: A novel epigenetic regulator of myeloid derived suppressor cell expansion and function. Mol. Immunol. 63, 579-585. doi: 10.1016/j.molimm.2014.08.002

Seto, E., and Yoshida, M. (2014). Erasers of histone acetylation: the histone deacetylase enzymes. Cold Spring Harb. Perspect. Biol. 6:a018713. doi: 10.1101/ cshperspect.a018713

Shao, D., Villet, O., Zhang, Z., Choi, S. W., Yan, J., Ritterhoff, J., et al. (2018). Glucose promotes cell growth by suppressing branched-chain amino acid degradation. Nat. Commun. 9:2935.

Shao, J., Weng, X., Zhuo, L., Yu, L., Li, Z., Shen, K., et al. (2019). Angiotensin II induced CSF1 transcription is mediated by a crosstalk between different epigenetic factors in vascular endothelial cells. Biochim. Biophys. Acta Gene Regul. Mech. 1862, 1-11. doi: 10.1016/j.bbagrm.2018.10.001

Tampe, B., and Zeisberg, M. (2014). Evidence for the involvement of epigenetics in the progression of renal fibrogenesis. Nephrol. Dialy. Transplant. 29(Suppl. 1), i1-i8. doi: 10.1093/ndt/gft361

Tikoo, K., Sharma, E., Amara, V. R., Pamulapati, H., and Dhawale, V. S. (2016). Metformin improves metabolic memory in high fat diet (HFD)-induced renal dysfunction. J. Biol. Chem. 291, 21848-21856. doi: 10.1074/jbc.c116.732990

Voelter-Mahlknecht, S., Ho, A. D., and Mahlknecht, U. (2005). Chromosomal organization and localization of the novel class IV human histone deacetylase 11 gene. Intern. J. Mol. Med. 16, 589-598.

Wang, H., Cheng, F., Woan, K., Sahakian, E., Merino, O., Rock-Klotz, J., et al. (2011). Histone deacetylase inhibitor LAQ824 augments inflammatory responses in macrophages through transcriptional regulation of IL-10. J. Immunol. 186, 3986-3996. doi: 10.4049/jimmunol.1001101

Watanabe, Y., Khodosevich, K., and Monyer, H. (2014). Dendrite development regulated by the schizophrenia-associated gene FEZ1 involves the ubiquitin proteasome system. Cell Rep. 7, 552-564. doi: 10.1016/j.celrep.2014. 03.022

Weng, X., Zhang, Y., Li, Z., Yu, L., Xu, F., Fang, M., et al. (2019). Class II transactivator (CIITA) mediates IFN-gamma induced eNOS repression by enlisting SUV39H1. Biochim. Biophys. Acta Gene Regul. Mech. 1862, 163-172. doi: 10.1016/j.bbagrm.2019.01.005

Xiong, C., Guan, Y., Zhou, X., Liu, L., Zhuang, M. A., Zhang, W., et al. (2019). Selective inhibition of class IIa histone deacetylases alleviates renal fibrosis. FASEB J. 33, 8249-8262. doi: 10.1096/fj.201801067rr

Xu, H., Wu, X., Qin, H., Tian, W., Chen, J., Sun, L., et al. (2015). Myocardinrelated transcription factor a epigenetically regulates renal fibrosis in diabetic nephropathy. J. Am. Soc. Nephrol. 26, 1648-1660. doi: 10.1681/asn.2014070678

Xu, Z., Li, W., Han, J., Zou, C., Huang, W., Yu, W., et al. (2017). Angiotensin II induces kidney inflammatory injury and fibrosis through binding to myeloid differentiation protein-2 (MD2). Sci. Rep. 7:44911.

Yang, Y., Li, X., Peng, L., An, L., Sun, N., Hu, X., et al. (2018). Tanshindiol C inhibits oxidized low-density lipoprotein induced macrophage foam cell formation via a peroxiredoxin 1 dependent pathway. Biochim. Biophys. Acta Mol. Basis Dis. 1864, 882-890. doi: 10.1016/j.bbadis.2017.12.033
Yang, Y., Liu, L., Fang, M., Bai, H., and Xu, Y. (2019a). The chromatin remodeling protein BRM regulates the transcription of tight junction proteins: Implication in breast cancer metastasis. Biochim. Biophys. Acta Gene Regul. Mech. 1862, 547-556. doi: 10.1016/j.bbagrm.2019.03.002

Yang, Y., Liu, L., Li, M., Cheng, X., Fang, M., Zeng, Q., et al. (2019b). The chromatin remodeling protein BRG1 links ELOVL3 trans-activation to prostate cancer metastasis. Biochim. Biophys. Acta Gene Regul. Mech. 1862, 834-845. doi: 10.1016/j.bbagrm.2019.05.005

Yanginlar, C., and Logie, C. (2018). HDAC11 is a regulator of diverse immune functions. Biochim. Biophys. Acta Gene Regul. Mech. 1861, 54-59. doi: 10.1016/ j.bbagrm.2017.12.002

Yu, L., Li, Z., Fang, M., and Xu, Y. (2017). Acetylation of MKL1 by PCAF regulates pro-inflammatory transcription. Biochim. Biophys. Acta Gene Regul. Mech. 1860, 839-847. doi: 10.1016/j.bbagrm.2017.05.006

Yu, L., Yang, G., Zhang, X., Wang, P., Weng, X., Yang, Y., et al. (2018). Megakaryocytic Leukemia 1 (MKL1) bridges epigenetic activation of NADPH Oxidase in macrophages to cardiac ischemia-reperfusion injury. Circulation 138, 2820-2836. doi: 10.1161/circulationaha.118.035377

Zeisberg, E. M., Tarnavski, O., Zeisberg, M., Dorfman, A. L., McMullen, J. R., Gustafsson, E., et al. (2007). Endothelial-to-mesenchymal transition contributes to cardiac fibrosis. Nat. Med. 13, 952-961.

Zeisberg, M., and Neilson, E. G. (2010). Mechanisms of tubulointerstitial fibrosis. J. Am. Soc. Nephrol. 21, 1819-1834. doi: 10.1681/asn.2010080793

Zeng, S., Wu, X., Chen, X., Xu, H., Zhang, T., and Xu, Y. (2018). Hypermethylated in cancer 1 (HIC1) mediates high glucose induced ROS accumulation in renal tubular epithelial cells by epigenetically repressing SIRT1 transcription. Biochim. Biophys. Acta Gene Regul. Mech. 1861, 917-927. doi: 10.1016/j. bbagrm.2018.08.002

Zhang, D., Li, H., Jiang, X., Cao, L., Wen, Z., Yang, X., et al. (2017). Role of AP-2alpha and MAPK7 in the regulation of autocrine TGF-beta/miR-200b signals to maintain epithelial-mesenchymal transition in cholangiocarcinoma. J. Hematol. Oncol. 10:170.

Zhang, X., Liu, S., Weng, X., Wu, T., Yu, L., Xu, Y., et al. (2018a). Brgl transactivates endothelium-derived colony stimulating factor to promote calcium chloride induced abdominal aortic aneurysm in mice. J. Mol. Cell. Cardiol. 125, 6-17. doi: 10.1016/j.yjmcc.2018.10.012

Zhang, X., Liu, S., Weng, X., Zeng, S., Yu, L., Guo, J., et al. (2018b). Brg1 deficiency in vascular endothelial cells blocks neutrophil recruitment and ameliorates cardiac ischemia-reperfusion injury in mice. Int. J. Cardiol. 269, 250-258. doi: 10.1016/j.ijcard.2018.07.105

Zhang, Y., Yuan, Y., Li, Z., Chen, H., Fang, M., Xiao, P., et al. (2019). An interaction between BRG1 and histone modifying enzymes mediates lipopolysaccharideinduced proinflammatory cytokines in vascular endothelial cells. J. Cell. Biochem. 120, 13216-13225. doi: 10.1002/jcb.28595

Zhou, B., Zeng, S., Li, N., Yu, L., Yang, G., Yang, Y., et al. (2017). Angiogenic factor with $G$ patch and FHA domains 1 is a novel regulator of vascular injury. Arterioscler. Thromb. Vasc. Biol. 37, 675-684. doi: 10.1161/atvbaha.117.308992

Conflict of Interest: The authors declare that the research was conducted in the absence of any commercial or financial relationships that could be construed as a potential conflict of interest.

Copyright (c) 2020 Mao, Liu, Zhang, Qin, Wu and Xu. This is an open-access article distributed under the terms of the Creative Commons Attribution License (CC BY). The use, distribution or reproduction in other forums is permitted, provided the original author(s) and the copyright owner(s) are credited and that the original publication in this journal is cited, in accordance with accepted academic practice. No use, distribution or reproduction is permitted which does not comply with these terms. 